\title{
Calogero-Sutherland system with two types interacting spins
}

\author{
S. Kharchev ${ }^{b}$ A. Levin ${ }^{\natural}$ M. Olshanetsky ${ }^{b}$ A. Zotov $\diamond b$ \\ b - Institute of Theoretical and Experimental Physics, Moscow, 117218, Russia \\ $\natural$ - International Laboratory for Mirror Symmetry and Automorphic Forms, \\ Mathematics Department of NRU HSE, Usacheva str. 6, Moscow, 119048, Russia \\ $\diamond$ - Steklov Mathematical Institute RAS, Gubkina str. 8, Moscow, 119991, Russia \\ $\S$ - Institute for Information Transmission Problems RAS (Kharkevich Institute), \\ Bolshoy Karetny per. 19, Moscow, 127994, Russia
}

Emails: kharchev@itep.ru, alevin2@hse.ru, olshanet@itep.ru,zotov@mi.ras.ru

\begin{abstract}
We consider the classical Calogero-Sutherland system with two types of interacting spin variables. It can be reduced to the standard Calogero-Sutherland system, when one of the spin variables vanishes. We describe the model in the Hitchin approach and prove complete integrability of the system by constructing the Lax pair and the classical $r$-matrix with the spectral parameter on a singular curve.
\end{abstract}

Introduction. The Calogero-Sutherland (CS) model [1, 2, 3] describes one-dimensional system of interacting pairwise particles through long range potentials. It has a lot of applications, in particular with the quantum Hall effect [4], matrix models [5], and orthogonal polynomials [6]. In this paper we consider the classical case. The classical CS model is an integrable system in the Liouville sense. Moreover, it remains integrable if one adds the so-called spin variables. The resulting system has form of the Euler-Arnold SL $(N)$ top with the inertia tensor depending on the positions of interacting particles [7].

Denote the coordinates of the particles $\mathbf{u}=\left(u_{1}, \ldots, u_{N}\right)$, their momenta $\mathbf{v}=\left(v_{1}, \ldots, v_{N}\right)$ and the spin variables $\left\{S_{j k}\right\}$ arranged into matrix $S=\sum_{i j}^{N} E_{i j} S_{i j}$ (here $\left\{E_{i j}\right\}$ is the standard basis in $\operatorname{Mat}(N)$, i.e. $\left.\left(E_{i j}\right)_{a b}=\delta_{i a} \delta_{b j}\right)$. The latter is an element of the Lie algebra $\operatorname{sl}(N)$. The spin CS model is described by the Hamiltonian

$$
H^{C S}=\frac{1}{2} \sum_{j=1}^{N} v_{j}^{2}-\sum_{j<k} \frac{S_{j k} S_{k j}}{\sinh ^{2}\left(u_{j}-u_{k}\right)} .
$$

The Poisson brackets between positions of particles and momenta are canonical $\left\{v_{k}, u_{j}\right\}=\delta_{j k}$, while the Poisson structure for $\left\{S_{j k}, S_{m n}\right\}$ is given by the Dirac brackets. They can be obtained starting from the Lie-Poisson brackets on the Lie coalgebra $\mathrm{sl}^{*}(N)$ after imposing constraints $S_{\text {diag }}=0$ (and some gauge fixation) resulting from the coadjoint action of the diagonal subgroup of $\operatorname{SL}(N)$ on the spin variables $S$. The case when $S \in \operatorname{so}(N)$ is know as well [8]. Some further generalizations can be found in [9]. 
Summary. Our generalization of (1) is as follows:

$$
H=\frac{1}{2} \sum_{j=1}^{N} v_{j}^{2}+\sum_{j<k} \frac{S_{j k}^{2}+T_{j k}^{2}-2 S_{j k} T_{j k} \cosh \left(u_{j}-u_{k}\right)}{\sinh ^{2}\left(u_{j}-u_{k}\right)},
$$

where $S_{i j}, T_{i j}$ are elements of antisymmetric matrices $S$ and $T\left(S_{j k}=-S_{k j}, T_{j k}=-T_{k j}\right)$ with the Lie-Poisson brackets on the direct sum of two Lie coalgebras $\operatorname{so}^{*}(N) \oplus \operatorname{so}^{*}(N)$ :

$$
\begin{gathered}
\left\{S_{i j}, S_{k l}\right\}=-\frac{1}{2}\left(S_{i l} \delta_{k j}-S_{k j} \delta_{i l}-S_{i k} \delta_{l j}+S_{l j} \delta_{i k}\right), \\
\left\{T_{i j}, T_{k l}\right\}=\frac{1}{2}\left(T_{i l} \delta_{k j}-T_{k j} \delta_{i l}-T_{i k} \delta_{l j}+T_{l j} \delta_{i k}\right), \\
\left\{S_{i j}, T_{k l}\right\}=0 .
\end{gathered}
$$

The phase space $\mathbb{R}^{2 N-2} \times \mathcal{O}_{\mathrm{SO}(N)} \times \mathcal{O}_{\mathrm{SO}(N)}$ consists of $\mathbb{R}^{2 N-2}$ parameterized by momenta and positions of $N$ particles in the center of mass frame and two coadjoint orbits $\mathcal{O}_{\mathrm{SO}(N)}$. Each orbit is obtained from $\mathrm{so}^{*}(N)$ by fixation of the Casimir functions (i.e. the eigenvalues of matrices $S$ and T). The Poisson structure (3) keeps the same form on $\mathcal{O}_{\mathrm{SO}(N)} \times \mathcal{O}_{\mathrm{SO}(N)}$. The dimension of generic $\mathrm{SO}(N)$ orbit equal. $1 / 2)\left(N^{2}-N\right)-[N / 2]$. Therefore, the dimension of the total phase space is $(N-1)(N+2)-2[N / 2]$.

For $N=2$ the Lie algebra so(2) is commutative and the spin variables are fixed. In this case we obtain from (2) the Hamiltonian with two constants

$$
H=\frac{v^{2}}{2}+\frac{m_{1}^{2}+m_{2}^{2}-2 m_{1} m_{2} \cosh (2 u)}{\sinh ^{2}(2 u)},
$$

which reproduces the $\mathrm{CS}$ model of the $\mathrm{BC}_{1}$ type 3 .

We prove that there exists $(1 / 2)(N-1)(N+2)-[N / 2]$ independent integrals of motion in involution, and the Hamiltonian (2) is one of them. To this end we construct the Lax pair and the classical $r$-matrix. Similarly to $\operatorname{so}(N)$ version of (10) 8] (and in contrast to the $\operatorname{sl}(N)$ spin CS system) the $M$-operator can be explicitly constructed because the spin variables $S, T$ are skew-symmetric matrices, and the additional reduction is not needed.

We derive the generalized CS system (GCS) using the Hitchin approach [10, 11]. The Lax operator of integrable system satisfies the Hitchin equations. They come from the self-duality equations in four dimension after their reduction to two dimensional Riemann surface. Namely, instead of $\mathbb{R}^{4}$ one consider the four-dimensional space $\mathbb{R}^{2} \times \Sigma$, where $\Sigma$ plays the role of the base spectral curve 2 . The field content of the Hitchin system comes from the four-dimensional vector-potentials independent on the first two coordinates. One of them is the Higgs field that plays the role of the Lax operator of the integrable system. The coordinates of particles describe the moduli of solutions of the Hitchin equations, while the spin variables are the residues of the Higgs fields at the singular points. On Fig.1 and Fig.2 (see the last page) the base spectral curves $\Sigma$ of CS and GCS systems are depicted. The Hitchin systems on singular curve (and, in particular, CS system) were studied previously in [12, 13].

Another important ingredient of the our construction is the so-called quasi-compact structure of the gauge group. It means that the gauge transformations at the singular points on the base spectral curve are reduced to the unitary group 3 . We will come to this structure in relation to integrable systems elsewhere. As a result the spin variables become elements of the unitary algebra $\mathrm{su}(\mathrm{N})$. To come to the integrable case we further reduce them to the orthogonal algebra so(N).

\footnotetext{
${ }^{1}[x]$ stands for integer part of $x$.

${ }^{2}$ The spectral curve of integrable system is defined as characteristic equation for the Lax matrix. It is a branched covering of the base spectral curve, where the spectral parameter lives.

${ }^{3}$ In the standard approach to the Hitchin systems the gauge group may have the quasi-parabolic structure, i.e. the gauge group is reduced at singular points to the triangular subgroup.
} 
Hitchin system structure. The described system is the Hitchin system over a singular curve. The base spectral curve:

The curve is defined in the following way. Consider two rational curves $\Sigma_{\alpha} \sim \mathbb{C} P^{1},(\alpha=1,2)$ with corresponding holomorphic coordinates $z_{1}, z_{2} \in \mathbb{C}_{1,2}$ Fig.2. They are glued at the points $z_{1}^{0}=z_{2}^{0}=0$ and $z_{1}^{\infty}=z_{2}^{\infty}=\infty$. The singular curve is $\Sigma=\Sigma_{1} \cup \Sigma_{2}$ with this identification.

The field content:

1. The anti-holomorphic vector potentials $\bar{A}_{\alpha}\left(z_{\alpha}, \bar{z}_{\alpha}\right)$ on the components $\Sigma_{\alpha}$ taking values in the Lie algebra $\operatorname{sl}(N, \mathbb{C})$;

2. The Higgs fields $\Phi_{\alpha}\left(z_{\alpha}, \bar{z}_{\alpha}\right)$ are holomorphic 1-forms on $\Sigma_{\alpha} \backslash\{0,1, \infty\}$ taking values in the Lie algebra $\operatorname{sl}(N, \mathbb{C})$. They have simple poles at $z_{\alpha}=0,1, \infty$ with the definite residues. Let $T$ be an element of the Lie algebra $\operatorname{su}(N), \eta \in \operatorname{sl}(N, \mathbb{C}), h \in \mathrm{SU}(N)$ and $g \in \mathrm{SL}(N, \mathbb{C})$. Then we assume that

$$
\begin{array}{lll}
\text { 1. } & \left.\operatorname{Res} \Phi_{\alpha}\left(z_{\alpha}=1\right)\right|_{s u(N)}=0, & \\
& z_{\alpha}=0 & z_{\alpha}=\infty \\
\text { 2. } & \left.\operatorname{Res} \Phi_{1}\right|_{s u(N)}=T, & \left.\operatorname{Res} \Phi_{1}\right|_{s u(N)}=\left.\eta\right|_{s u(N)}, \\
\text { 3. } & \left.\operatorname{Res} \Phi_{2}\right|_{s u(N)}=h T h^{-1}, & \left.\operatorname{Res} \Phi_{2}\right|_{s u(N)}=\left.g \eta g^{-1}\right|_{s u(N)} .
\end{array}
$$

These variables $\bar{A}_{\alpha}, \Phi_{\alpha}$ form the Higgs bundle $\mathcal{H}_{\mathrm{SL}}(N, \mathbb{C})(\Sigma)$ over the curve $\Sigma$.

Symplectic structure and symplectic reduction:

$\mathcal{H}_{\mathrm{SL}}(N, \mathbb{C})(\Sigma)$ can be considered as an infinite-dimensional symplectic manifold. It is equipped with the symplectic form

$$
\begin{gathered}
\omega=\sum_{\alpha=1,2} \int_{\Sigma_{\alpha}} D\left\langle\Phi_{\alpha}, D \bar{A}_{\alpha}\right\rangle+D \int_{\Sigma_{1}}\left(\left.\delta\left(z_{1}, \bar{z}_{1}\right)\right|_{z_{1}=\infty}\left\langle\eta, g^{-1} D g\right\rangle+\left.\delta\left(z_{1}, \bar{z}_{1}\right)\right|_{z_{1}=0}\left\langle T, h^{-1} D h\right\rangle\right) \\
+D \int_{\Sigma_{2}}\left(\left.\delta\left(z_{2}, \bar{z}_{2}\right)\right|_{z_{2}=\infty}\left\langle\eta, g^{-1} D g\right\rangle+\left.\delta\left(z_{2}, \bar{z}_{2}\right)\right|_{z_{2}=0}\left\langle T, h^{-1} D h\right\rangle\right)
\end{gathered}
$$

where $\langle$,$\rangle is the Killing form on \operatorname{sl}(N, \mathbb{C})$. The gauge symmetries of the system are symplectic transformations preserving (6). They are formed by the pair of smooth maps $f_{\alpha} \in \Sigma_{\alpha} \in$ $C^{\infty}\left(\Sigma_{\alpha}\right) \rightarrow \operatorname{SL}(N, \mathbb{C})$ such that at the fixed point

$$
\left.f_{\alpha}\left(z_{\alpha}, \bar{z}_{\alpha}\right)\right|_{z_{\alpha}=0,1, \infty} \in S U(N) .
$$

The gauge action on the dynamical variables is

$$
\begin{gathered}
\bar{\partial}_{\alpha}+\bar{A}_{\alpha} \rightarrow f_{\alpha}\left(\bar{\partial}_{\alpha}+\bar{A}_{\alpha}\right) f_{\alpha}^{-1}, \\
g \rightarrow f_{2}(\infty) g f_{1}^{-1}(\infty), f_{\alpha}(\infty)=\left.f_{\alpha}\left(z_{\alpha}, \bar{z}_{\alpha}\right)\right|_{z_{\alpha}=\infty}, \\
h \rightarrow f_{2}(0) h f_{1}^{-1}(0), f_{\alpha}(0)=\left.f_{\alpha}\left(z_{\alpha}, \bar{z}_{\alpha}\right)\right|_{z_{\alpha}=0}, \\
\Phi_{\alpha} \rightarrow f_{\alpha} \Phi_{\alpha} f_{\alpha}^{-1},\left.\quad T \rightarrow f_{1} T f_{1}^{-1}\right|_{z_{1}=0},\left.\quad \eta \rightarrow f_{1} \eta f_{1}^{-1}\right|_{z_{1}=\infty} .
\end{gathered}
$$

where, according with (7), in (9) $f_{1}, f_{2} \in \mathrm{SU}(N)$. The gauge transformations generate the moment maps

$$
\begin{gathered}
\mu_{1}=\partial_{\bar{z}_{1}} \Phi_{1}+\left.\left.\delta\left(z_{1}, \bar{z}_{1}\right)\right|_{z_{1}=\infty} \eta\right|_{s u(N)}+\left.\delta\left(z_{1}, \bar{z}_{1}\right)\right|_{z_{1}=0} T \\
\mu_{2}=\partial_{\bar{z}_{2}} \Phi_{2}+\left.\left.\delta\left(z_{2}, \bar{z}_{2}\right)\right|_{z_{2}=\infty}\left(g \eta g^{-1}\right)\right|_{s u(N)}+\left.\delta\left(z_{2}, \bar{z}_{2}\right)\right|_{z_{2}=0} S,\left(S=h T h^{-1}\right) .
\end{gathered}
$$

We put the Gauss law constraints $\mu_{1}=0, \mu_{2}=0$. This means that the Higgs fields $\Phi_{\alpha}\left(z_{\alpha}, \bar{z}_{\alpha}\right)$ are meromorphic on $\Sigma_{\alpha}$ and have simple unitary poles at $z_{\alpha}=0,1, \infty$ with the prescribed residues. The generic configuration of the vector potentials $\bar{A}_{\alpha}$ can be gauged away $\left(\bar{A}_{\alpha}=0\right)$ by the gauge group action (8). The residual gauge transformations $\mathcal{G}_{1}^{\text {res }}$ are only constants $\mathcal{G}_{1}^{\text {res }}=\left\{f_{\alpha} \in S U(N)\right\}$. 
For generic $g$ the transformation (9) allows one to diagonalize $g$

$$
f_{2}(\infty) g f_{1}^{-1}(\infty)=\exp (\mathbf{u}), \quad \mathbf{u}=\operatorname{diag}\left(u_{1}, \ldots, u_{N}\right), \quad u_{j} \in \mathbb{R} .
$$

Thus, the variables $(h, T, \mathbf{u}, \mathbf{v})$ describe the reduced phase space, where $\mathbf{v}$ is a variable dual $\mathbf{u}$ coming from diagonal part of $\Phi_{\alpha}$. In this way after reduction we come to the finite-dimensional phase space $\tilde{\mathcal{R}}^{\text {red }}$. It has dimension $\operatorname{dim} \tilde{\mathcal{R}}^{\text {red }}=2\left(N^{2}-1\right)+2(N-1)$.

The gauge transformations are defined up to the action from the right by the final residual gauge transformation $\mathcal{G}_{2}^{\text {res }}$ preserving the diagonal form of $g$ : $\mathcal{G}_{2}^{\text {res }}=f_{\alpha}(\infty) \rightarrow f_{\alpha}(\infty) s^{-1}$, $s \in \mathcal{G}_{2}^{\text {res }}=\mathcal{T} \rtimes W$, where $W$ is the Weyl group and $\mathcal{T}$ is the Cartan torus in $\mathrm{SU}(N)$. It implies that:

1. $\mathbf{u}$ can be ordered as $u_{1}>u_{2}>\ldots u_{N}$.

2. The element $h$ in (13) is defined up to the action $h \rightarrow s h s^{-1}, s \in \mathcal{G}_{2}^{r e s}=\mathcal{T} \rtimes W$. Imposing the corresponding constraints we come to the symplectic quotient $\mathcal{R}^{\text {red }}=\tilde{\mathcal{R}}^{\text {red }} / /(\mathcal{T} \rtimes W)$, $\operatorname{dim} \mathcal{R}^{\text {red }}=2\left(N^{2}-1\right)$.

Lax matrix. The gauge transformed Higgs fields $\Phi_{\alpha}=f_{\alpha} L_{\alpha} f_{\alpha}^{-1}$, where $f_{\alpha}$ is such that $\left.f_{\alpha}\left(z_{\alpha}\right)\right|_{z_{\alpha}=\infty}$ diagonalize $g$, will play the role of the Lax operators. The following form of the Lax operators has the correct structure of poles

$$
L_{1}\left(z_{1}\right)=\frac{T}{z_{1}}+\frac{T+\eta}{1-z_{1}}, \quad L_{2}\left(z_{2}\right)=\frac{S}{z_{2}}+\frac{S+g \eta g^{-1}}{1-z_{2}}, \quad\left(S=h T h^{-1}\right)
$$

if

$$
\left\{\begin{array}{c}
T+\left.\eta\right|_{s u(N)}=0 \\
S+\left.\left(g \eta g^{-1}\right)\right|_{s u(N)}=0
\end{array}\right.
$$

cf. (5). The solution of these equations assumes the form:

$$
\begin{gathered}
\eta=P+S_{\text {diag }}+T_{\text {diag }}+X, P=\operatorname{diag} \mathbf{v}=\operatorname{diag}\left(v_{1}, \ldots, v_{N}\right), \\
X_{j k}=\frac{S_{j k}-T_{j k} \exp \left(-u_{j k}\right)}{2 \sinh \left(u_{j k}\right)}, \quad S_{k j}=-\bar{S}_{j k}, \quad u_{j k}=u_{j}-u_{k}, j<k .
\end{gathered}
$$

The integrals of motion $I_{l k}(k=0, \ldots l, l=2, \ldots, N)$ come from the expansion

$$
\begin{gathered}
\left\langle(T+\eta)^{l}\right\rangle=\left\langle T^{l}\right\rangle+l\left\langle T^{l-1} \eta\right\rangle+\ldots+\left\langle\eta^{l}\right\rangle, \\
I_{l k}=\left\langle T^{l-k} \eta^{k}\right\rangle, \quad(k=0, \ldots, l) .
\end{gathered}
$$

In particular, $H$ (2) is $\frac{1}{2} I_{2,2}$. Notice that the integrals $I_{l, 0}$ are the Casimir function of the Lie-Poisson algebra on $\operatorname{sl}(N, \mathbb{C})^{*}$. Excluding the number of $l$ Casimir functions we obtain the number of integrals

$$
\mathcal{N}_{G}=\sum_{l=2}^{N}(l+1)-(N-1)=\frac{1}{2}(N-1)(N+2) .
$$

Evidently, all integrals are functionally independent. But for the complete integrability in $\mathrm{su}(N)$ case we need $\mathcal{N}_{G}=\frac{1}{2} \operatorname{dim}_{\mathbb{R}}\left(\mathcal{R}^{\text {red }}\right)=N^{2}-1$, so we have less integrals of motion than needed.

$\mathbf{S O}(N)$ model. To go around it we pass from the Lie algebra $\operatorname{su}(N)$ to $\operatorname{so}(N)$ for the spin variables $S, T$. It means that $T_{j k}, S_{j k} \in \mathbb{R}, S_{j k}=-S_{k j}, T_{j k}=-T_{k j}\left(S_{\text {diag }}=T_{\text {diag }}=0\right)$ and the eigenvalues of $S, T$ are fixed. As we explained after (3), the dimension of the phase space $\operatorname{dim}_{\mathbb{R}}\left(\mathcal{R}^{r e d}\right)=(N-1)(N+2)-2[N / 2]$ in this case, and the number of integrals $\mathcal{N}_{G}(19)$ is even more than needed. It happens because in $\mathrm{SO}(N)$ case the Hamiltonians $I_{l 1}$ from (18) for even values of $l$ (in the interval $2 \leq l \leq N$ ) turn into the Casimir functions (of matrix $T$ ). This follows 
from the skew-symmetry of matrices $S, T$ and can be verified by direct substitution. Finally, the number of integrals of motion $\mathcal{N}_{G}{ }^{\prime}=\mathcal{N}_{G}-[N / 2]$ is equal to the half of dimension of the phase space: $\frac{1}{2} \operatorname{dim}_{\mathbb{R}}\left(\mathcal{R}^{\text {red }}\right)=\mathcal{N}_{G}{ }^{\prime}=\frac{1}{2}(N-1)(N+2)-[N / 2]$.

Let us define the Lax equation on one of the components $\Sigma_{\alpha}$ of the base spectral curve $\Sigma$. Consider the following Lax pair:

$$
\begin{gathered}
L_{i j}(z)=\delta_{i j} v_{i}+\left(1-\delta_{i j}\right)\left(\frac{S_{i j}}{\sinh \left(u_{i j}\right)}-\left(\operatorname{coth}\left(u_{i j}\right)+\operatorname{coth}(z)\right) T_{i j}\right)=\tilde{\eta}_{i j}-(1+\operatorname{coth}(z)) T_{i j}, \\
M_{i j}=\left(1-\delta_{i j}\right) \frac{T_{i j}-S_{i j} \cosh \left(u_{i j}\right)}{\sinh ^{2}\left(u_{i j}\right)} .
\end{gathered}
$$

The Lax matrix (20) is related to $L_{1}\left(z_{1}\right)$ (14) by $L(z)=\left(1-z_{1}\right) L_{1}\left(z_{1}\right), \operatorname{coth}(z)=-\left(1+z_{1}\right) / z_{1}$ with simultaneous rescaling $S \rightarrow 2 S$ and $T \rightarrow 2 T$ (i.e. $\tilde{\eta}=P+2 X$ in contrast to $\eta=P+X$ ). The latter is just a convenient way to avoid unnecessary numerical coefficients. Finally, the Lax equation

$$
\dot{L}(z):=\{H, L(z)\}=[L(z), M]
$$

with the Lax pair (20), (21) provides equations of motion generated by the Hamiltonian (2) and the Poisson structure given by (3) together with the canonical Poisson brackets $\left\{v_{j}, u_{k}\right\}=\delta_{j k}$. Indeed, the Lax equation (22) is equivalent to

$$
\dot{\tilde{\eta}}=[\tilde{\eta}, M], \quad \text { where } \quad \tilde{\eta}_{i j}=\delta_{i j} v_{i}+\left(1-\delta_{i j}\right) \frac{S_{i j}-T_{i j} \exp \left(-u_{i j}\right)}{\sinh \left(u_{i j}\right)}
$$

and

$$
\dot{T}=[T, M] .
$$

From (24) we have

$$
\dot{T}_{i j}=\sum_{k \neq i, j} T_{i k} T_{k j}\left(\frac{1}{\sinh ^{2}\left(u_{k j}\right)}-\frac{1}{\sinh ^{2}\left(u_{i k}\right)}\right)-T_{i k} S_{k j} \frac{\cosh \left(u_{k j}\right)}{\sinh ^{2}\left(u_{k j}\right)}+S_{i k} T_{k j} \frac{\cosh \left(u_{i k}\right)}{\sinh ^{2}\left(u_{i k}\right)}
$$

Substitution of (25) into (24) provides equations of motion for $S$ and $\mathbf{v}, \mathbf{u}$ variables:

$$
\begin{gathered}
\dot{u}_{i}=v_{i}, \quad \dot{v}_{i}=\sum_{k \neq i} 2 \frac{\cosh \left(u_{i k}\right)}{\sinh ^{3}\left(u_{i k}\right)}\left(S_{i k}^{2}+T_{i k}^{2}\right)-2 \frac{\cosh ^{2}\left(u_{i k}\right)+1}{\sinh ^{3}\left(u_{i k}\right)} S_{i k} T_{i k}, \\
\dot{S}_{i j}=\sum_{k \neq i, j} S_{i k} S_{k j}\left(\frac{1}{\sinh ^{2}\left(u_{i k}\right)}-\frac{1}{\sinh ^{2}\left(u_{k j}\right)}\right)+S_{i k} T_{k j} \frac{\cosh \left(u_{k j}\right)}{\sinh ^{2}\left(u_{k j}\right)}-T_{i k} S_{k j} \frac{\cosh \left(u_{i k}\right)}{\sinh ^{2}\left(u_{i k}\right)} .
\end{gathered}
$$

It is straightforward to verify that (25), (26) and (27) are generated by the Hamiltonian (2)).

Classical r-matrix. The $r$-matrix structure is given by

$$
\left\{L_{1}(z), L_{2}(w)\right\}=\left[L_{1}(z), r_{12}(z, w)\right]-\left[L_{2}(w), r_{21}(w, z)\right]
$$

with $s o(N) r$-matrix found in [8] for $\mathrm{SO}(N)$ spin Calogero model:

$$
\begin{gathered}
r_{12}(z, w)=\frac{1}{2}(\operatorname{coth}(z-w)+\operatorname{coth}(z+w)) \sum_{i} E_{i i} \otimes E_{i i}+ \\
\frac{1}{2} \sum_{i \neq j} E_{i j} \otimes E_{j i}\left(\operatorname{coth}(z-w)+\operatorname{coth}\left(u_{i j}\right)\right)+\frac{1}{2} \sum_{i \neq j} E_{i j} \otimes E_{i j}\left(\operatorname{coth}(z+w)+\operatorname{coth}\left(u_{i j}\right)\right)
\end{gathered}
$$

The proof of (28) is also straightforward. Being written in the form (28) the Poisson brackets provide the involutivity of the integrals of motion (18). One can also verify that $r$-matrix (29) provides $M$-matrix (21) via

$$
\operatorname{tr}_{2}\left(r_{12}(z, w) L_{2}(w)\right)=\frac{1}{2}(\operatorname{coth}(z-w)+\operatorname{coth}(z+w)) L(z)-M
$$


Acknowledgements This work was funded by the Russian Science Foundation (RSCF) grant 16-12-10344.

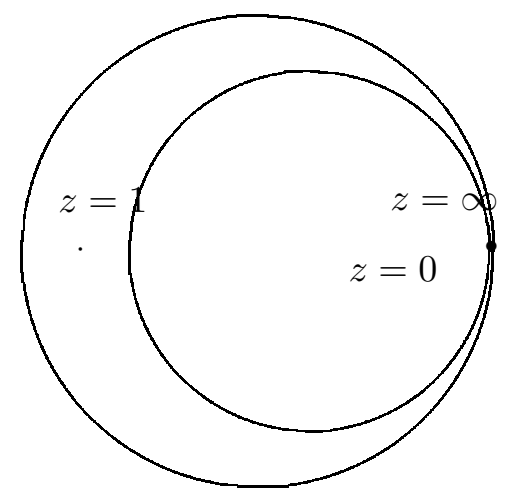

Fig.1 Spectral curve for CS system

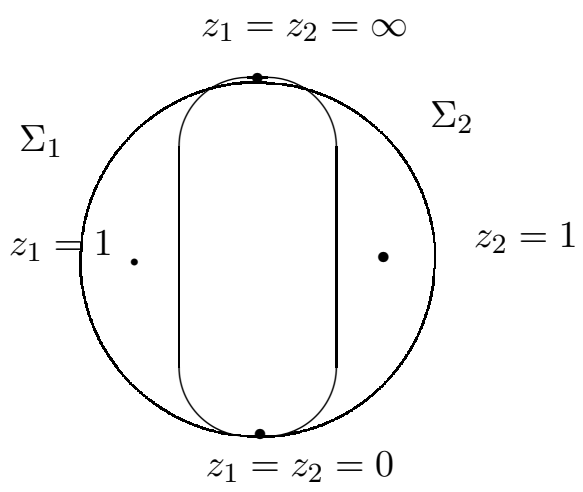

Fig.2 Spectral curve for generalized CS system

\section{References}

[1] F. Calogero, J. Math. Phys. 10 (1969) 2191-2196.

[2] B. Sutherland, Physical Review A, 4:5 (1971) 2019-2021.

B. Sutherland, Physical Review A, 5:3 (1972) 1372-1376.

[3] M.A. Olshanetsky, A.M. Perelomov, Inventiones mathematicae, 37:2 (1976) 93-108.

[4] H. Azuma, S. Iso, Physics Letters B, 331 (1994) 107-113.

[5] H. Awata, Y. Matsuo, S. Odake, J. Shiraishi, Physics Letters B, 347 (1995) 49-55.

[6] T.H. Baker, P.J. Forrester, Commun. Math. Phys., 188 (1997) 175-216.

[7] J. Gibbons, T. Hermsen, Physica D: Nonlinear Phenomena 11 (1984) 337-348;

S. Wojciechowski, Physics Letters A, 111 (1985) 101-103.

[8] E. Billey, J. Avan, O. Babelon, Physics Letters A, 188:3 (1994) 263-271.

[9] L. Fehér, B.G. Pusztai, Nuclear Physics B, 734 [FS] (2006) 304-325.

[10] N.J. Hitchin, Proceedings of the London Mathematical Society, 3 (55) (1987) 59-126.

[11] N. Hitchin, Duke Math. J., 54:1 (1987), 91-114.

[12] N. Nekrasov, Commun. Math. Phys., 180 (1996), 587-603.

[13] D.V. Talalaev, A.V. Chervov, Theoret. and Math. Phys, 140:2 (2004) 1043-1072. 